\title{
Perfil de ácidos grasos de aceite de Sacha inchi (Plukenetia volubilis) en comparación con otros aceites vírgenes comestibles
}

\author{
Fatty acid profile of sacha inchi (Plukenetia volubilis) oil in \\ comparison to other virgin edible oils
}

Recibido: julio abril de 2016 | Revisado: mayo de 2016 | Aceptado: junio de 2016

\author{
FERNANDo RAmos-EsCUdero ${ }^{1}$ \\ Lady Ponce Dayer ${ }^{1}$ \\ Edy BARNETT MENDOZA ${ }^{2}$ \\ Luis Celi SAavedra ${ }^{2}$ \\ Mónica Ramos Escudero 2
}

1 Laboratorio de Química Analítica, Escuela de Ingeniería en Industrias Alimentarias, Universidad de San Martín de Porres, Lima, Perú diomedes.fernando@gmail.com indalim23@gmail.com

2 Facultad de Ingeniería y Arquitectura, Escuela de Ingeniería en Industria Alimentarias, Universidad de San Martín de Porres, Lima, Perú ebarnettm@usmp.pe

lcelis@usmp.pe

mramos@usmp.pe

\begin{abstract}
The consumption of omega-3 fatty acids, either from animal or vegetable origin may protect against cardiovascular diseases. Sacha inchi oil is considered a promising crop for its healthy contributions, and from the point of view of fatty acids is still a majority compared to other virgin oils. The content of saturated fatty acids is of $7.04 \%$, while the content of polyunsaturated fatty acids is over 82.90. Among the closest oils we find chia oil with $81 \%$ PUFA, followed by Sacha inchi (Plukenetia huayllabambana) oil with $78 \%$, while flaxseed oil lies in fourth place with $70 \%$. The data suggests that consumption of Sacha inchi oil can be considered as a functional ingredient.
\end{abstract}

Key words: Sacha inchi oil, virgin edible oils, fatty acids

\section{Resumen}

El consumo de ácidos grasos omega-3 ya sea de origen animal o vegetal puede proteger de las enfermedades cardiovasculares. El aceite de Sacha Inchi se considera como un cultivo promisorio por su aporte saludable, además desde el punto de vista de ácidos grasos aún sigue siendo mayoritario en comparación con otros aceites vírgenes. El contenido de ácidos grasos saturados se encuentra alrededor de $7.04 \%$, mientras que el contenido de ácidos grasos poliinsaturados sobre $82.90 \%$. Entre los aceites más cercanos se encuentra el aceite de chía con $81 \%$ en PUFA, seguido del aceite de Sacha Inchi (Plukenetia huayllabambana) con $78 \%$, mientras que el aceite de linaza se ubica en la cuarta posición con $70 \%$. Estos datos sugieren que el consumo de aceite de Sacha Inchi puede ser considerado como un ingrediente funcional.

Palabras clave: aceite de Sacha Inchi, aceites vírgenes comestibles, ácidos grasos 


\section{Introducción}

Los aceites vegetales se obtienen a partir de una fuente vegetal, tales como verduras, frutos secos o semillas. Los aceites vegetales comestibles se utilizan principalmente en la cocina, como aderezos para ensaladas, en la fabricación de margarinas y pastas para untar, y en panadería. Durante los últimos 100-150 años, ha habido un crecimiento sustancial en la producción y el consumo de aceites vegetales, que dio lugar a un amplio debate sobre los posibles efectos para la salud. Diversos aceites vegetales tienen diferencias importantes en la composición de ácidos grasos y también diferentes composiciones en contenido no glicerídica incluyendo fenoles, esteroles, tocoferoles, y escualeno, pero estos nutrientes varían, sustancialmente, dependiendo de las condiciones de procesamiento y de almacenamiento (Savva \& Kafatos, 2016).

Los aceites comestibles son reconocidos como nutrientes esenciales en las die- tas tanto humanas como animales. Desde un punto de vista nutricional, son fuentes concentradas de energía que proporcionan ácidos grasos esenciales que se consideran como bloques de construcción para las hormonas, así como un medio de soporte para las vitaminas liposolubles A, D, E, y K (Ivanova-Petropulosa et al., 2015).

El consumo de aceites vegetales como alimento se pronostica una expansión del 3 por ciento en 2016/17, lo que refleja el crecimiento de la población y el PIB. Los aceites vegetales se espera que sigan creciendo principalmente, los aceites de soja y palma que cuentan aproximadamente con el $63 \%$ de la producción mundial de aceites vegetales (Foreign Agricultural Service - United States Departament of Agriculture [FAS-USDA], 2016). En la Tabla 1 , se muestra la producción mundial de aceites comestibles de diferentes fuentes vegetales.

Tabla 1

Principales aceites vegetales: suministro y distribución mundial

\begin{tabular}{lcccc}
\hline Producción & $2012 / 13$ & $2013 / 14$ & $2014 / 15$ & $2015 / 16$ \\
\hline Aceite de coco & 3.72 & 3.45 & 3.44 & 3.49 \\
Aceite de algodón & 5.22 & 5.17 & 5.13 & 4.42 \\
Aceite de oliva & 2.48 & 3.14 & 2.37 & 2.90 \\
Aceite de palma & 56.38 & 59.34 & 61.64 & 61.55 \\
Aceite de maní & 5.48 & 5.70 & 5.52 & 5.43 \\
Aceite de colza & 25.69 & 27.26 & 27.64 & 27.34 \\
Aceite de soya & 43.10 & 45.11 & 49.04 & 52.23 \\
Aceite de girasol & 12.88 & 15.55 & 14.96 & 15.12 \\
\hline
\end{tabular}

Nota: Extraído de Oilseed world markets and trade. (FAS-USDA, 2016)

Los principales aceites vírgenes que se comercializan son los de oliva y girasol (aunque la mayor parte de este último es refinado), algunas de semillas (como el cártamo, colza, soja, pepitas de uva, de calabaza) o de algu- nos frutos secos (nuez, almendra, avellana) (Navas-Hernández, 2010). Por otro lado, el aceite de Sacha inchi emerge como recurso interesante por su riqueza nutricional. Debido a ello, este aceite ha sido investigado por 
su composición particular y sus beneficios potenciales para la salud.

Las semillas de Sacha Inchi (Plukenetia volubilis $\mathrm{L}$.) son consideradas una fuente rica de proteínas y aceite. El aceite se caracteriza por su alto contenido de ácidos grasos poliinsaturados principalmente de $\alpha$-linolénico (C18:3) y linoleico (C18:2), lo que representa $-82 \%$ del contenido total de aceite (Fanali et al., 2011). Otros componentes de interés nutricional son los fitoesteroles, tocoferoles, carotenos (Chirinos et al., 2013) y compuestos fenólicos (Fanali et al., 2011).

\section{Análisis de ácidos grasos}

En los últimos años, se ha prestado cada vez más atención a los aceites consumidos por los seres humanos. Los aceites vegetales más populares (Tabla 1) contienen importantes cantidades de ácidos grasos insaturados, sobre todo aquellos que son necesarios para el organismo humano (Ramos-Escudero, 2014). Sin embargo, en algunos casos, debido a la alta cantidad de ácidos grasos insaturados, estos aceites son susceptibles a cambios en su composición, que pueden producirse durante la cosecha de la materia prima, la producción del aceite, o durante el transporte y almacenamiento (Gromadzka y Wardencki, 2011).

Sobre la base de la expansión del mercado de aceites vegetales la verificación de la autenticidad y la trazabilidad de los aceites tienen que ser adecuados para la prevención de adulteraciones y de un etiquetado incorrecto deliberado o accidental. Por este motivo, la autenticidad se ha convertido en un tema importante tanto desde el punto de vista comercial como del aseguramiento de la salud pública (Aparicio, Conte y Fiebig 2013; Cserháti, Forgács, Deyl y Miksik, 2005). La fracción saponificable representa más del $98 \%$ del aceite y mayoritaría y está constituida por los componentes mayoritarios (Lerma-García, 2012; Morales y León-Camacho, 2003).

Los ácidos grasos son estructuras simples constituidas por cadenas largas de diferente número de átomos de carbono, con un grupo ácido carboxílico en un extremo. Además, las características de los aceites vegetales están influenciadas por la proporción de los ácidos grasos presentes y es uno de los principales indicadores de la calidad y frescura. Los ácidos grasos son los componentes mayoritarios de cualquier aceite comestible, para su determinación generalmente se convierten en ésteres metílicos de ácidos grasos (FAMEs).

Los ésteres metílicos se preparan por metilación, en un medio metanólico, que se puede llevar a cabo con álcali, ácido o catálisis alcalina y ácida (Aparicio y Aparicio-Ruíz, 2000).

La determinación de la composición de ácidos grasos en los aceites vegetales se lleva a cabo, por lo general, mediante cromatografía de gases (GC) con detector de ionización de llama (FID) (Cserháti et al., 2005).

En la Tabla 2, se muestran algunos de los análisis más comunes para determinar la pureza de los ácidos grasos. 
Tabla 2

Descripción general de algunos métodos empleados para determinar la autenticidad del aceite de Sacha inchi

\begin{tabular}{ccll}
\hline Asunto & Extracción del aceite & \multicolumn{1}{c}{ Analito } & \multicolumn{1}{c}{ Técnica } \\
\hline C & $\mathrm{P}$ & $\begin{array}{l}\text { ácidos grasos } \\
\text { ácido linolénico } \\
\text { ácido linoleico } \\
\text { ácido oleico }\end{array}$ & GC-FID \\
A & $\mathrm{P}$ & ácidos grasos & GC-FID \\
$\mathrm{C}$ & $\mathrm{P}$ & ácidos grasos & GC-FID \\
$\mathrm{C}$ & $\mathrm{SC}-\mathrm{CO}_{2}$ & ácidos grasos & GC-FID \\
$\mathrm{C}$ & $\mathrm{S}$ & ácidos grasos & GC-FID/MS \\
$\mathrm{C}$ & $\mathrm{S}$ & ácidos grasos & GC-FID \\
$\mathrm{C}$ & No indica & ácidos grasos & GC-FID \\
$\mathrm{C}$ & No indica & ácidos grasos & FTIR y ${ }^{1} \mathrm{H}$ NMR \\
$\mathrm{C}$ & $\mathrm{P}$ & ácidos grasos & GC-FID \\
$\mathrm{C}$ & $\mathrm{P}$ & ácidos grasos & GC-FID \\
$\mathrm{C}$ & $\mathrm{S}$ & $\mathrm{P}$ &
\end{tabular}

Nota: A, autentificación; C, caracterización; P, prensado; S, solvente; $\mathrm{SC}-\mathrm{CO}_{2}$, dióxido de carbono supercrítico; Regresión de mínimos cuadrados parciales (PLSR), cromatografía de gases-detector de ionización de llama (GC-FID); Espectroscopia infrarroja con reflectancia total atenuada (IR ATR); espectrofotometría de masas (MS); espectroscopia infrarroja con transformada de Fourier (FTIR); espectroscopia de resonancia magnética nuclear de protón ( $\left.{ }^{1} \mathrm{H} \mathrm{NMR}\right)$.

\section{Perfil de ácidos grasos de aceite de Sacha inchi}

La composición de ácidos grasos y la relación de las sumas y ratios del aceite de Sacha Inchi están resumidos en la Tablas 3 y 4, respectivamente.

Tabla 3

Composición de ácidos grasos del aceite de Sacha inchi (Plukenetia volubilis)

\begin{tabular}{|c|c|c|c|c|c|c|c|c|}
\hline \multirow{2}{*}{$\begin{array}{l}\text { Sacha } \\
\text { inchi }\end{array}$} & \multicolumn{7}{|c|}{ Ácidos grasos (\%) } & \multirow{2}{*}{ Referencias } \\
\hline & 12:0 & 14:0 & 16:0 & 18:0 & $18: 1$ & $18: 2$ & 18:3 & \\
\hline 1 & & & 4.7 & 3.5 & 10.7 & 33.5 & 44.0 & $\begin{array}{l}\text { Maurer, Hata-Sakoda, Pascual-Chagman } \\
\text { y Rodríguez-Saona, } 2012\end{array}$ \\
\hline 2 & & & 4.2 & 2.5 & 8.4 & 34.1 & 50.4 & $\begin{array}{l}\text { Follegatti-Romero, Pianto, Grimaldi y } \\
\text { Cabral, } 2009\end{array}$ \\
\hline 3 & & & 4.0 & 3.0 & 9.0 & 40.0 & 44.0 & Liu et al., 2014 \\
\hline 4 & & & 4.7 & 1.6 & 8.1 & 27.4 & 58.1 & Zuleta, Ríos y Benjumea, 2012 \\
\hline 5 & & & 4.0 & 3.0 & 9.0 & 36.0 & 47.0 & Fanali et al., 2011 \\
\hline
\end{tabular}

En la Tabla 3, se observa la composición de ácidos grasos de cinco muestras analizadas por diferentes autores. Como puede observarse los compuestos mayoritarios son ácidos grasos insaturados como el oleico $(\mathrm{C} 18: 1 \omega 9+\omega 7)$ y predominan principalmente ácido linoleico

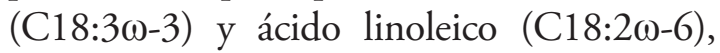
además los ácidos grasos saturados presentes mayoritarios encontrados son el palmítico (C16:0) y esteárico (C18:0). 
Tabla 4

Suma de ácidos grasos del aceite de Sacha inchi

\begin{tabular}{cccccc}
\hline \multirow{2}{*}{$\begin{array}{c}\text { Sacha } \\
\text { inchi }\end{array}$} & \multicolumn{5}{c}{ Ácidos grasos (\%) } \\
\cline { 2 - 6 } & $\Sigma$ SFA & $\Sigma$ MUFA & इPUFA & SFA/PUFA & L/L \\
\hline 1 & 8.2 & 10.7 & 77.5 & 0.11 & 0.76 \\
2 & 6.7 & 8.4 & 84.5 & 0.08 & 0.68 \\
3 & 7.0 & 9.0 & 84.0 & 0.08 & 0.91 \\
4 & 6.3 & 8.1 & 85.5 & 0.07 & 0.47 \\
5 & 7.0 & 9.0 & 83.0 & 0.08 & 0.77 \\
\hline
\end{tabular}

Nota: L/L, relación ácido linoleico/linolénico; SFA, total ácido grasos saturados; MUFA, total ácidos grasos monoinsaturados; PUFA, total ácidos grasos poliinsaturados y relación SFA/PUFA.

\section{Perfil de ácidos grasos de otros aceites vegetales}

En la Tabla 5, se muestra el perfil de ácidos grasos de diferentes aceites vírgenes co-

Tabla 5

Composición de ácidos grasos de aceites vírgenes comestibles

\begin{tabular}{|c|c|c|c|c|c|c|}
\hline \multirow{2}{*}{ Recursos } & \multicolumn{5}{|c|}{ Ácidos grasos (\%) } & \multirow{2}{*}{ Referencias } \\
\hline & $16: 0$ & 18:0 & $18: 1$ & $18: 2$ & $18: 3$ & \\
\hline Argán & 12.7 & 5.8 & 45.6 & 34.6 & 0.34 & Rueda et al., 2014 \\
\hline Chia & 7.1 & 3.4 & 7.0 & 18.2 & 62.8 & Marineli et al., 2014 \\
\hline Oliva $^{1}$ & 10.1 & 3.7 & 78.7 & 5.1 & 0.9 & $\begin{array}{l}\text { Ramos-Escudero, Morales y Asuero, } \\
2015\end{array}$ \\
\hline Linaza & 5.8 & 3.1 & 20.5 & 15.0 & 55.2 & Rueda et al., 2014 \\
\hline Soya & 9.2 & 3.8 & 24.4 & 54.0 & 8.0 & Rueda et al., 2014 \\
\hline Palta & 16.3 & 1.5 & 60.6 & 14.7 & 0.7 & Rueda et al., 2014 \\
\hline $\mathrm{Uva}^{2}$ & 8.1 & 4.5 & 20.5 & 66.5 & 0.4 & Navas-Hernández, 2010 \\
\hline Sésamo ${ }^{3}$ & 10.4 & 5.4 & 40.9 & 42.7 & 0.6 & Navas-Hernández, 2010 \\
\hline Sacha inchi ${ }^{4}$ & 6.6 & 3.8 & 9.4 & 26.7 & 51.3 & $\begin{array}{l}\text { Chirinos, Pedreschi, Domínguez y } \\
\text { Campos, } 2015\end{array}$ \\
\hline
\end{tabular}

${ }^{1}$ Variedad Picual; ${ }^{2 \text { y } 3}$ Semillas; ${ }^{4}$ Plukenetia huayllabambana

De los datos observados, en la Tabla 5, los aceites vírgenes comestibles que presentan mayor concentración de ácidos grasos poliinsaturados es el aceite de chia > aceite mestibles, entre ellos los de mayor impacto por sus bondades nutricionales y saludables como la chía, lino, argán y otros cultivos tradicionales y no tradicionales. 
Tabla 6

Suma de ácidos grasos de aceites vírgenes comestibles

\begin{tabular}{|c|c|c|c|c|c|}
\hline \multirow{2}{*}{ Recursos } & \multicolumn{5}{|c|}{ Ácidos grasos (\%) } \\
\hline & $\Sigma S F A$ & $\Sigma$ MUFA & $\Sigma$ PUFA & SFA/PUFA & $\mathrm{L} / \mathrm{L}$ \\
\hline Argán & 18.5 & 45.6 & 34.9 & 0.53 & 101.76 \\
\hline Chia & 10.5 & 7.0 & 81.0 & 0.13 & 0.29 \\
\hline Oliva $^{1}$ & 13.8 & 78.7 & 6.0 & 2.30 & 5.67 \\
\hline Linaza & 8.9 & 20.5 & 70.2 & 0.13 & 0.27 \\
\hline Soya & 13.0 & 24.4 & 62.0 & 0.21 & 6.75 \\
\hline Palta & 17.8 & 60.6 & 15.4 & 1.16 & 21.00 \\
\hline $\mathrm{Uva}^{2}$ & 12.6 & 20.5 & 66.9 & 0.19 & 166.25 \\
\hline Sésamo ${ }^{3}$ & 15.8 & 40.9 & 43.3 & 0.36 & 71.17 \\
\hline Sacha inchi ${ }^{4}$ & 10.4 & 9.4 & 78.00 & 0.13 & 0.52 \\
\hline
\end{tabular}

Nota: L/L, relación ácido linoleico/linolénico; SFA, total ácido grasos saturados; MUFA, total ácidos grasos monoinsaturados; PUFA, total ácidos grasos poliinsaturados y relación SFA/PUFA. ${ }^{1}$ Variedad Picual; ${ }^{2 \text { y } 3}$ Semillas; ${ }^{4}$ Plukenetia huayllabambana

\section{Discusión}

Los estudios realizados en muestras de Sacha inchi presentan contenidos de PUFAs mayores o iguales a $82.9 \%$ (Follegatti-Romero et al., 2009; Fanali et al., 2011; Zuleta et al., 2012; Maurer et al., 2012; Liu et al., 2014). En comparación con el aceite de chía que presenta un contenido de $81.0 \%$ (Marineli et al., 2014), mientras que el aceite de Sacha inchi (Plukenetia huayllabambana) alrededor de 78\% (Chirinos et al., 2015).

Por otro lado, es importante recalcar que la concentración de SFA es mayoritaria en el aceite de argán $>$ aceite de palta $>$ aceite de sésamo (Rueda et al., 2014; Navas-Hernández, 2010), mientras que el aceite de Sacha inchi (Plukenetia volubilis) se encuentra alrededor de $7.0 \%$.

\section{Conclusiones}

El aceite de Sacha inchi es un importante recurso de ácidos grasos poliinsaturados (omega 3) y componentes bioactivos. Es por esta razón quizás que el consumo de aceites vírgenes ricos en poliinsaturados como el aceite de Sacha inchi proporciona importantes beneficios para la salud de los seres humanos. El principal ácido graso es el $\alpha$-linolénico (C18:3 $\omega-3)$, y la suma del contenido de insaturados se encuentra entre alrededor de 90 a 93\%, el resto de los aceites vírgenes se encuentra por debajo de este valor. 


\section{Referencias}

Aparicio, R. \& Aparicio-Ruíz, R. (2000). Authentication of vegetable oils by chromatographic techniques. Journal of Chromatography $A, 881,93-104$.

Aparicio, R., Conte, L. S., \& Fiebig, H. J. (2013). Olive oil authentication. In R. Aparicio-Ruiz \& J. Harwood (Eds.), Handbook of olive oil: Analysis and properties (pp. 589-653) New York: Springer Science+Business.

Chirinos, C., Pedreschi, R., Domínguez, G. \& Campos, D. (2015). Comparison of the physico-chemical and phytochemical characteristics of the oil of two Plukenetia species. Food Chemistry, 173, 1203-1206.

Chirinos, R., Zuloeta, G., Pedreschi, R., Mignolet, E., Larondelle, Y. \& Campos, D. (2013). Sacha inchi (Plukenetia volubilis): A seed source of polyunsaturated fatty acids, tocopherols, phytosterols, phenolic compounds and antioxidant capacity. Food Chemistry, 141, 1732-1739.

Cserháti, T., Forgács, E., Deyl, Z., \& Miksik, I. (2005). Chromatography in authenticity and traceability tests of vegetable oils and dairy products: a review. Biomedical Chromatography, 19, 183-190.

Fanali, C., Dugo, L., Cacciola, F., Beccaria, M., Grasso, S., Dachà, M. \& Mondello, L. (December, 2011). Chemical characterization of Sacha inchi (Plukenetia volubilis L.) oil. Journal of Agricultural and Food Chemistry, 59(24), 13043-13049. doi: 10.1021/ jf203184y

Follegatti-Romero L. A., Piantino, C. R., Grimaldi, R. \& Cabral, F. A. (2009). Supercritical CO2 extraction of omega-3 rich oil from Sacha inchi (Plukenetia volubilis L.) seeds. Journal of Supercritical Fluids, 49, 323-329.

Foreign Agricultural Service - United States Departament of Agriculture (2016).
Orlseeds: world Markets and Trade. Recuperado de www.fas.usda.gor/ data/otlseeds-world-markets-an-trade.

Gromadzka, J. \& Wardencki, W. (2011). Trends in edible vegetable oils analysis. Part A. Determination of different components of edible oils-a review. Polish Journal Food Nutrition Sciences, 61, 33-43.

Ivanova-Petropulos, V., Mitrev, S., Stafilov, T., Markova, N., Leitner, E., Lankmayr, E. \& Siegmund, B. (2015). Characterisation of traditional Macedonian edible oils by their fatty acid composition and their volatile compounds. Food Research International, 77, 506-514.

Lerma-García, M. J. (2012). Characterization and authentication of olive and other vegetable oils. In author (Ed.), Introduction: Edible oils (pp. 1-43). Berlin: Springer-Verlag.

Liu, Q., Xu, Y. K., Zhang, P., Na, Z., Tang, T. \& Shi, Y. X. (2014). Chemical composition and oxidative evolution of Sacha inchi (Plukentia volubilis L.) oil from Xishuangbanna (China). Grasas y Aceites, 65, 1-9.

Marineli, R. S., Moraes, E. A., Lenquiste, S. A., Godoy, A. T., Eberlin, M. N. \& Maróstica, M. R. (2014). Chemical characterization and antioxidant potential of Chilean chia seeds and oil (Salvia hispánica L.). LWT-Food Science and Technology, 59, 1304-1310.

Maurer, N. E, Hatta-Sakoda, B., Pascual-Chagman, G. \& Rodriguez-Saona, L.E. (2012). Characterization and authentication of a novel vegetable source of omega-3 fatty acids, Sacha inchi (Plukenetia volubilis L.) oil. Food Chemistry, 134, 1173-1180.

Morales, M. T. y León-Camacho, M. (2003). Cromatografía de gases y líquidos: metodología aplicada al aceite de oliva. En R. Aparicio y J. Harwood 
(Eds.), Manual del aceite de oliva (pp. 163-207). Madrid: Mundi-Prensa.

Navas-Hernández, P. B. (2010). Componentes minoritarios y propiedades antioxidantes de aceites vírgenes y tortas residuales obtenidos por presión en frio a partir de fuentes vegetales convencionales y no convencionales (Tesis de Doctorado). Universidad de Castilla La Mancha, Ciudad Real, España.

Ramos-Escudero, F. (2014). Caracterización y trazabilidad del aceite de Sacha inchi (Plukenetia volubilis Linneo) (Tesis de Doctorado). Universidad de Sevilla, Sevilla, España.

Ramos-Escudero, F., Morales, M. T. \& Asuero, A. G. (2015). Characterization of bioactive compounds from monovarietal virgin olive oils: Relationship between phenolic compounds-antioxidant capacities. International Journal of Food Properties, 18, 348-358.

Rueda, A., Seiquer, I., Olalla, M., Giménez, R., Lara, L. \& Cabrera-Vique, C. (2014). Characterization of fatty acid profile of argan oil and other edible vegetable oils by gas chromatography and discriminant analysis. Journal of Chemistry, 2014, 1-8.

Savva, S. C. \& Kafatos, A. (2016). Vegetable oils: Dietary importance. Reference Module in Food Science, 365-372.

Zuleta, E. C., Rios, L. A. \& Benjumea, P. N. (2012). Oxidative stability and cold flow behavior of palm, sacha-inchi, jatropha and castor oil biodiesel blends. Fuel Processing Technology, 102, 96-101. 